\title{
The Red Dwarf Star Population in the Galaxy
}

\author{
L.V. Mirzoyan \\ Byurakan Astrophysical Observatory, Armenia
}

Red dwarf stars in the Galaxy occur in three forms: as flare stars, $T$ Tau stars and red dwarf stars of constant brightness. Haro (1957) suggested that all these stars present regular evolutionary stages of red dwarf stars.

The space distribution of UV Cet flare stars in the solar vicinity indicates that these low luminosity stars belong to the disk population of the Galaxy. Therefore one can suppose that all red dwarf stars have a space distribution which is similar to that of the flare stars (Mirzoyan et al. 1988a). Only a small part of them is found in star clusters and associations. The T Tau stars are an exception: because of their very short lifetime, all of them are found in stellar associations.

The space distribution of red dwarf stars is determined by the fact that all of them are formed in star clusters and associations and are finally lost from their stellar systems and merge into the general galactic field (Mirzoyan 1995).

The total number of flare stars can be estimated under the assumption that the mean density of these stars in the general galactic field is equal to that in the solar vicinity. The density of UV Cet stars in a sphere of radius $20 \mathrm{pc}$ around the Sun is about $4 \cdot 10^{-3} \mathrm{pc}^{-3}$ (Gershberg 1978), but this is a lower limit. Taking $10^{-2} \mathrm{pc}^{-3}$, and $250 \mathrm{pc}$ for the thickness of the galactic disk, the total number of flare stars in the Galaxy is $5.6 \cdot 10^{8}$ (Mirzoyan et al. 1988b).

The total number of red flare stars in the Galaxy can be estimated from the percentage of flare stars among all red dwarfs. Using the Catalogue of Nearby Stars (Gliese 1969, Gliese \& Jahreiss 1979), and the catalogue of UV Cet stars (Gershberg 1978), this percentage is estimated for the red dwarf stars of spectral classes $K$ and $M$ (Mirzoyan et al. 1989). Table 1 shows the distribution of this percentage for red dwarfs in absolute magnitude bins $\left(M_{\mathrm{pg}}\right)$, where $N$ is the total number of red dwarfs and $N_{\mathrm{UV}}$ the number of flare stars among them.

A regular increase of the percentage towards fainter magnitudes is indicated. It can be supposed that the bright red dwarf stars in the galactic field have already terminated their flare activity, while the stars of lower luminosities have kept their ability to produce flares until today.

Table 1 shows that the total number of red dwarf stars in the solar neighborhood is larger than that of flare stars by about one order of magnitude. Therefore the total number of red dwarf stars in the Galaxy, including the total number of 
Table 1. Percentage of UV Cet type flare stars among the near red dwarf stars of spectral classes $\mathrm{K}$ and $\mathrm{M}$ (Mirzoyan et al. 1989)

\begin{tabular}{crrc}
\hline \multicolumn{1}{c}{$M_{\mathrm{PB}}$} & \multicolumn{1}{c}{$N$} & $N_{\mathrm{UV}}$ & $N_{\mathrm{UV}} / N(\%)$ \\
\hline $7.5-8.5$ & 50 & 1 & 2 \\
$8.5-9.5$ & 100 & 3 & 3 \\
$9.5-10.5$ & 131 & 7 & 5 \\
$10.5-11.5$ & 67 & 10 & 15 \\
$>11.5$ & 113 & 47 & 42 \\
\hline Total & 461 & 68 & 15 \\
\hline
\end{tabular}

stars which are found today in star clusters and associations, is $\approx 10^{10}$ (Mirzoyan et al. 1988b). This is in agreement with the estimate of the total number of stars, which originated in stellar associations during the lifetime of the Galaxy $\left(10^{10}\right.$ years), supposing that the star formation process had a constant intensity.

Ambartsumian (1950) showed that the total number of stars that originated in associations in the Galaxy for $10^{10}$ years is about $10^{9}$ in OB-associations, and more than $10^{10}$ in $\mathrm{T}$-associations. Since all stars that originated in $\mathrm{T}$-associations and the overwhelming majority of those that originated in OB-associations are red dwarf stars, the total number of red dwarf stars is at least $10^{10}$. We do not take into account the red dwarf stars of spectral classes $F$ and $G$, which must be mainly in the general galactic field. This does not change our order-of-magnitude estimate.

The masses of red dwarf stars are from a few $M_{\odot}$ for the T Tau stars to some hundredths for the flare stars of lowest luminosities. When taking $0.1-1.0 M_{\odot}$ as the mean mass of red dwarf stars, their total mass in the Galaxy is of the order of $10^{9}-10^{10} M_{\odot}$, which does not contradict Oort's (1958) estimate.

Thus, the space distribution of red dwarf stars in the Galaxy corresponds to their ages: the stars are found in 'maternal' systems if they are young enough, and in the general galactic field, if they are older.

\section{References}

Ambartsumian V.A., 1950, Izv. Acad. Sci. USSR Ser. Phys. 14, 15

Gershberg R.E., 1978, Flare Stars of Small Masses, Nauka, Moscow

Gliese W., 1969, Veröff. Astr. Rechen-Inst. Heidelberg, No. 22

Gliese W., Jahreiss H., 1979, A\&AS 38, 423

Haro G., 1957, in IAU Symp. No. 3, Non-Stable Stars, G.H. Herbig (ed.), Cambridge University Press, Cambridge, p. 26

Mirzoyan L.V., 1995, this volume, p. 47

Mirzoyan L.V., Hambarian V.V., Garibjarian A.T., Mirzoyan A.L., 1988a, Astrofiz. 29,44

Mirzoyan L.V., Hambarian V.V., Garibjanian A.T., Mirzoyan A.L., 1988b, Astrofiz. 29,531

Mirzoyan L.V., Hambarian V.V., Garibjanian A.T., Mirzoyan A.L., 1989, Astrofiz. 31, 259

Oort J.H., 1958, in Stellar Populations, D.J.K. O'Connell, S.J. (ed.), North Holland, Amsterdam, p. 25 